\title{
Clock synchronization, a universal light speed, and the terrestrial redshift experiment
}

\author{
Alan Macdonald \\ Department of Mathematics \\ Luther College, Decorah, IA 52101, U.S.A. \\ macdonal@luther.edu
}

Am. J. Phys. 51, 795 (1983)

\begin{abstract}
This paper (i) gives necessary and sufficient conditions that clocks in an inertial lattice can be synchronized, (ii) shows that these conditions do not imply a universal light speed, and (iii) shows that the terrestrial redshift experiment provides evidence that clocks in a small inertial lattice in a gravitational field can be synchronized.
\end{abstract}

The central, and revolutionary, postulate of special relativity is that the speed of light between two points in an inertial frame is a universal constant. The speed is defined in terms of synchronized clocks at the points. (See below.) But the Hafele-Keating experiment [1] and muon decay experiments which measure time dilation [2] show that a universal time does not exist, and so the notion of separated synchronized clocks can have no a priori meaning. It follows that the speed of light can have no meaning until a definition of synchronized clocks is given. It is not simply that the speed cannot be measured; it can have no meaning.

The purpose of this paper is not to enter into the debate surrounding the above argument, for I believe that its logic is sound. Instead, the purpose is to accept the conclusion of the argument and provide a proper foundation for the universal light speed postulate by giving a simple account of the physical principles involved in clock synchronization.

Some of the ideas developed here could profitably be used by teachers of relativity. I know of no special relativity text which discusses clock synchronization before the speed of light. The texts usually begin with the Michelson-Morley experiment and the universal light speed postulate and then show, among other things, that there is no universal time. An alert student can be confused and ask: If there is no universal time, then what was meant in the first place by the speed of light? An approach starting with the Hafele-Keating experiment, the nonexistence of a universal time, and clock synchronization avoids this logical problem. (Another advantage of starting with the Hafele-Keating experi- 
ment is that it is a much more direct and dramatic violation of intuition than the Michelson-Morley experiment, the interpretation of which usually involves the introduction of a soon-to-be-abandoned ether, analogies with swimmers in rivers, discussion of constructive and destructive interference of light, etc.)

\section{Clock Synchronization}

By an inertial frame we mean an inertial cubical lattice made of rigid rods and with clocks at the nodes synchronized according to the following definition. (An accelerometer can determine that the lattice is inertial.) Emit a flash of light from a node $O$ to a node $P$ at time $t_{O}$ according to the clock at $O$. Let it arrive at $P$ at time $t_{P}$ according to the clock at $P$. Similarly, let a flash emitted from $P$ at time $t_{P}^{\prime}$ arrive at $O$ at $t_{O}^{\prime}$. Say the clocks are synchronized if

$$
t_{P}-t_{O}=t_{O}^{\prime}-t_{P}^{\prime}
$$

The definition is essentially due to Einstein [3].

We now show that the following two conditions are necessary and sufficient that clocks in an inertial lattice can be synchronized, or, in P. W. Bridgeman's descriptive phrase, that we can "spread time over space".

(i) Emit flashes of light from a node $R$ at times $t_{R}^{1}$ and $t_{R}^{2}$ according to a clock at $R$. Let them arrive at a node $S$ at times $t_{S}^{1}$ and $t_{S}^{2}$ according to the clock at $S$. Then

$$
t_{S}^{2}-t_{R}^{2}=t_{S}^{1}-t_{R}^{1}
$$

(ii) The time it takes light to traverse a triangle in the lattice is independent of the direction taken around the triangle.

The condition (i) states that the two sides of Eq. (1) do not depend on the times that the flashes are sent. It is a necessary and sufficient condition that two given clocks can be synchronized. For if the clocks can be synchronized, then Eq. (2) must hold in order that they remain synchronized. Conversely, assume Eq. (2) holds. Suppose the flash from $P$ to $O$ in Eq. (2) is the reflection of the flash from $O$ to $P$. Let $2 T=t_{O}^{\prime}-t_{O}$ be the round trip time. Then Eq. (2) can be written

$$
t_{P}=t_{O}+T
$$

the clocks are synchronized if the flash arrives at $P$ in half the time it takes for the round trip. If we write $2 T=\left(t_{O}^{\prime}-t_{P}\right)+\left(t_{P}-t_{O}\right)$, then Eq. (2) shows that $T$ is independent of the time the flash is sent. Now send a flash from $O$ to $P$ at any time $t_{O}$. When it arrives at $P$ set the clock there to $t_{O}+T$. According to Eq. (3) the clocks are synchronized.

Synchronize all clocks with the one at $O$ in this way.

We now show that (ii) is a necessary and sufficient condition that clocks at nodes $P$ and $Q$ are synchronized with each other once they are both synchronized

with the clock at $O$. The condition has been verified for a square to one part in $10^{12}[4]$. 
Reflect a flash of light around the triangle $O P Q$. Let the flash be at $O, P, Q, O$ at times $t_{O}, t_{P}, t_{Q}, t_{R}$ according to the clock at the node. Similarly, let the times for a flash sent around in the other direction be $t_{O}^{\prime}, t_{P}^{\prime}, t_{Q}^{\prime}, t_{R}^{\prime}$. We have the algebraic identities

$$
\begin{gathered}
t_{R}-t_{O}=\left(t_{R}-t_{Q}\right)+\left(t_{Q}-t_{P}\right)+\left(t_{P}-t_{O}\right) \\
t_{R}^{\prime}-t_{O}^{\prime}=\left(t_{R}^{\prime}-t_{P}^{\prime}\right)+\left(t_{P}^{\prime}-t_{Q}^{\prime}\right)+\left(t_{Q}^{\prime}-t_{O}^{\prime}\right) .
\end{gathered}
$$

Since the clock at $O$ is synchronized with those at $P$ and $Q$,

$$
t_{P}-t_{O}=t_{R}^{\prime}-t_{P}^{\prime}, \quad t_{R}-t_{Q}=t_{Q}^{\prime}-t_{O}^{\prime} .
$$

Thus the middle terms on the right-hand side of Eq. (4) are equal if, and only if, the left sides are equal, i.e., the clocks at $P$ and $Q$ are synchronized if and only if the condition (ii) is true for the triangle $O P Q$.

It follows from all this that conditions (i) and (ii) are necessary and sufficient conditions that clocks in an inertial lattice can be synchronized.

\section{Universal Light Speed}

In this section we assume that clocks in an inertial lattice have been synchronized. Let $P$ and $Q$ be nodes in the lattice at distance $D$ apart. Emit a flash of light from $P$ toward $Q$ at time $t_{P}$ according to the clock at $P$. Let it arrive at $Q$ at time $t_{Q}$ according to the clock at $Q$. Define the one-way speed of the light from $P$ to $Q$ by $D /\left(t_{Q}-t_{P}\right)$. This one-way speed requires two clocks, and to be meaningful, the clocks must be synchronized. If the flash is reflected at $Q$ back to $P$, arriving there at time $t_{P}^{\prime}$, define the two-way speed of the light from $P$ to $Q$ by $2 D /\left(t_{P}^{\prime}-t_{P}\right)$. This two-way speed requires one clock and so is independent of clock synchronization.

According to Eq. (1), if clocks at $P$ and $Q$ are synchronized then the oneway speed of light from $P$ to $Q$ is the same as the one-way speed from $Q$ to $P$. We emphasize that the equality of these speeds is a matter of definition which can neither confirmed nor refuted by experiment.

Conditions (i) and (ii), necessary and sufficient for clock synchronization, are easily verified for the classical Newtonian spacetime. But the two-way speed of light in this spacetime is different in perpendicular directions (except in the ether frame). This is precisely what the Michelson-Morely experiment was designed to detect. According to the above remarks, once clocks are synchronized in this space-time according to Eq. (1), the one-way speed of light will be different in perpendicular directions. Thus, despite claims to the contrary [5], the ability to synchronize clocks according to our definition does not imply a universal light speed. [Clocks synchronized in a Newtonian space-time according to Eq. (1) are not "really" synchronized. But this does not affect the logic of the argument.]

The Michelson-Morley and Kennedy-Thorndike [6] experiments show directly that the two-way speed of light is the same in different directions, in 
different inertial frames, and at different times. With clocks synchronized according to Eq. (1), we can interpret the experiments as showing that the one-way speed of light is the same in different directions, in different inertial frames, and at different times. This is the great advantage of the definition of synchronized clocks used here over all others. Conversely, the results of these experiments provide strong motivation for our definition of synchronized clocks: if the twoway speed of light has always the same value, what could be more natural than to define synchronized clocks so that the one-way speed has always this value?

\section{Terrestrial Redshift Experiment}

Local inertial frames are natural coordinates to use in a curved space-time. We now investigate the possibility of synchronizing clocks in a small inertial lattice in a gravitational field to form a local inertial frame.

A rearrangement of Eq. (2) gives

$$
t_{R}^{2}-t_{R}^{1}=t_{S}^{2}-t_{S}^{1} .
$$

The left-hand side is the time between the emission of the flashes at $R$ and the right side is the time between the reception of the flashes at $S$. If a clock at $R$ emits flashes at regular intervals to $S$, then Eq. (5) states that an observer at $S$ sees (actually sees) the clock at $R$ going at the same rate as his clock. Of course, the observer at $S$ will see all physical processes at $R$ proceeding at the same rate that they do at $S$.

If the "flashes" of light in Eq. (2) are successive wavecrests of light, then Eq. (5) states that the frequency of the received light is the same as that of the emitted light. Thus the condition (i), necessary and sufficient that two given clocks can be synchronized, is equivalent to a zero redshift between the points. The terrestrial redshift experiment [7] shows that this condition fails for points at the top and bottom of a tower. It follows that clocks in a small lattice at rest on the ground cannot be synchronized.

We now show that the experiment provides evidence that Eq. (2) does hold in a small inertial lattice in a gravitational field. In this way, the experiment provides evidence that clocks in a small inertial lattice in a gravitational field can be synchronized. In the experiment, the tower has (upward) acceleration $g$ in a small inertial lattice falling radially toward Earth. A simple calculation shows that the redshift in this tower having acceleration $g$ in an inertial lattice in the Earth's gravitational field is the same as the red shift in a tower having acceleration $g$ in an inertial frame in a flat spacetime [8]. Thus it is reasonable to assume that the redshift in a tower at rest in a small inertial lattice in a gravitational field is the same as the redshift in a tower at rest in an inertial frame. In other words, it is reasonable to assume that Eq. (2) (no redshift) holds in a small inertial lattice in a gravitational field. (It is desirable to test this directly be performing the experiment in orbit.) If we attribute this "proper" behavior of light in a small inertial lattice to no relative acceleration of the light and lattice, then we may say (loosely, but picturesquely) that the experiment 
provides evidence that light accelerates the same as matter in a gravitational field.

\section{References}

[1] J. C. Hafele and R. E. Keating, Science 77, 166, 168 (1972). Hafele and Keating brought two atomic clocks together, synchronized them, flew them around the Earth in opposite directions, reunited them, and found that they were no longer synchronized.

[2] J. Bailey et al., Nature 268, 301 (1977). In this experiment, the half-life of muons moving close to the speed of light is lengthened.

[3] A. Einstein, Ann. Phys. (Leipzig) 17, 1905. A translation appears in The Principle of Relativity (Dover, New York, 1952).

[4] W. M. Macek and D. T. M. Davis, Jr., Appl. Phys. Lett. 2, 67 (1963). In the experiment light was circulated in both directions around a square ring laser, setting up a standing wave. If $t_{1}$ and $t_{2}$ are the times for the light to travel around the square in the two directions and $f_{1}$ and $f_{2}$ are the frequencies in the two directions, then $t_{1} f_{1}=t_{2} f_{2}$, both sides being the number of wavelengths in the standing wave. When the laser was not rotating, $f_{1}$ and $f_{2}$ differed by no more than one part in $10^{12}$, showing that $t_{1}$ and $t_{2}$ differed by no more than one part in $10^{12}$.

[5] S. Fung and K. R. Hsieh, Am. J. Physics. 48, 654 (1980); H. Arzelies, Relativistic Kinematics (Pergamon, New York, 1966), p. 19; R. Demartins, Am. J. Phys. 50, 799 (1982).

[6] E. F. Taylor and J. A. Wheeler, Spacetime Physics (Freeman, San Francisco, 1966).

[7] R. V. Pound and J. L. Snider, Phys. Rev. Lett. 13, 539 (1964). In the experiment gamma radiation emitted at the bottom of the tower was redshifted when received at the top.

[8] We calculate the redshift in a tower having acceleration $g$ in an inertial frame. Suppose the tower is momentarily at rest when radiation is emitted from the bottom of the tower. The radiation travels a distance $h$, the height of the tower, in the inertial frame. (We ignore the small distance the tower moves during the flight of the radiation. We also ignore relativistic corrections to the calculation to follow. These effects are far too small to be detected by the experiment.) Thus the radiation takes time $z=g t=g h / c$. This speed causes a redshift $z=v / c=g h / c^{2}$. Within the accuracy of the experiment, this is the redshift observed. 